\title{
Bildung als Bürgerrecht oder: wozu Bildung?
}

\author{
Educação como cidadania ou: para quê educação?
}

\section{Zusammenfassung}

Der Text befasst sich mit dem Schlüsselbegriff der deutschen Pädagogik: "Bildung". Einleitend werden dessen semantische Unschärfe und Doppeldeutigkeit benannt sowie terminologische Abgrenzungen zu den Nachbarbegriffen "Ausbildung" und "Erziehung" vorgenommen. Als ein wesentliches Strukturmerkmal von Bildung wird anschließend dessen Prozesscharakter herausgestellt. Bildung ist ein chronokratisches, auf Zeit und Zeitlichkeit beruhendes Phänomen, was an konkreten Beispielen (subjektiv: Lernzeit, objektiv: Schulzeiten) erläutert wird. Bildung ist überdies ein topographisches Phänomen, da es an subjektiven (An-)Ordnungen und objektiven Räumlichkeiten gebunden ist; auch dieses Strukturmerkmal wird exemplarisch konkretisiert (Lebensweltentwürfe, Schulhausarchitektur). Nach diesen formalen Bestimmungen von Bildung wird die Problematik der Zielperspektive von Bildung aufgeworfen. Dies geschieht anhand der Frage nach dem Wozu von Bildung. Ausgehend vom klassischen, neuhumanistischen Verständnis, das den Selbstzweck von Bildung (Zweck an sich) betonte, entwickelt der Vortrag ein gesellschaftspolitisch und demokratisch ausgerichtetes Bildungskonzept, das auf das von den Soziologen Ralf Dahrendorf postulierte Bürgerrecht auf Bildung hinausläuft. Auf zwei Leitziele zugespitzt wird die Frage nach dem Wozu der Bildung beantwortet: die Leitziele lauten Glück (gelingendes Leben) und Gerechtigkeit (Moralität).

Schlüsselwörter: Bildung. Bürgerrecht. Gerechtigkeit. Glück. Lernzeit. Zetlichkeit.

\section{Resumo}

O presente texto ocupa-se com o conceito nuclear da pedagogia alemã, a saber, a formação. De maneira introdutória, são nomeadas suas imprecisões e ambiguidades semânticas, bem como as delimitações terminológicas em relação aos seus conceitos vizinhos, como "instrução" "educação". Como sinal estrutural essencial da formação, é apresentado seu caráter processual. Formação é um fenômeno cronocrático que, referindo-se ao tempo e à temporalidade, é esclarecido por meio de exemplos concretos: subjetivamente como tempo de aprendizagem; objetivamente como tempo escolar. Além disso, é um fenômeno topográfico que está ligado às ordenações subjetivas e às espacialidades objetivas. Essa característica estrutural também se encontra concretizada, exemplarmente, como projetos de vida e arquitetura do prédio escolar. Após essas determinações formais da noção de formação, é desenvolvida a problemática da finalidade de suas perspectivas. Isso acontece vinculado à pergunta sobre "para que formação?". Partindo da compreensão neo-humanista clássica, que acentua o fim intrínseco à formação (seu fim em si), expõe-se o conceito de formação orientado social, política e democraticamente. Tal exposição apoia-se na postulação de cidadania para a formação feita pelo sociólogo alemão Ralf Dahrendorf. A resposta para a pergunta "para que formação?" é dada com base em duas acentuadas finalidades principais: felicidade como vida bem-sucedida e justiça como moralidade.

Palavras-chave: Formação. Cidadania. Felicidade. Justiça. Tempo de aprendizagem. Temporalidade.

Recebido em 05/04/2017 - Aprovado em 19/07/2017

http://dx.doi.org/10.5335/rep.v24i3.7762

Promotion in Philosophie, Universität Kassel. Professor und Forscher an der Pädagogischen Hochschule in Karlsruhe, Deutschland. E-mail: hoyer@ph-karlsruhe.de 


\section{Einleitung}

Seit der Wende vom 18. zum 19. Jahrhundert ist Bildung, neben Erziehung, der Schlüsselbegriff in der deutschen Pädagogik (vgl. KOSELLECK, 2010, S. 105ff.). Aber er ist ein ziemlich unscharfer, schwer auf einen semantischen Nenner zu bringender Ausdruck. Was allerdings auf alle umkämpften Begriffe wie Glück, Gerechtigkeit, Wahrheit, Liebe, Moral etc. zutrifft, die eine so lange und bedeutungsvolle Geschichte haben.

\section{Ein Chamäleon unter den Begriffen}

Manche nennen Bildung einen Container- oder Passepartout-Begriff, da er je nach Vorliebe und Geisteshaltung mit verschiedenen Inhalten aufzufüllen sei. Man könnte auch von einem Begriffs-Chamäleon sprechen, weil das Wort in Abhängigkeit vom jeweiligen theoretischen Kontext seine Bedeutung wechselt. Mit dem Ausdruck ließe sich ein so sonderbarer Satz bilden wie: Bildung entsteht durch Bildung. Das hört sich wie eine sinnlose Tautologie an, so als würde man behaupten: Arbeitslosigkeit entsteht durch Arbeitslosigkeit oder Langeweile entsteht durch Langeweile. Der Satz ist nicht besonders aussagekräftig, aber sinnlos ist er nicht.

\section{Doppeldeutigkeit des Begriffs}

Dass der Satz "Bildung entsteht durch Bildung" ungeschickt, aber nicht sinnwidrig ist, ist der prinzipiellen Doppeldeutigkeit des Bildungsbegriffs geschuldet. Bildung kann sowohl einen Vorgang, einen individuellen Prozess als auch das Ziel dieses Vorgangs, einen Zustand, ein Ideal, eine Norm bezeichnen. Im Alltagssprachgebrauch (und auch in wissenschaftlichen oder politischen Texten) ist häufig unklar oder zumindest nicht auf Anhieb ersichtlich, ob mit Bildung ein Sein, ein bestimmtes Gut, oder ein Werden, eine Entwicklung, oder beides gemeint ist.

\section{Zur zeit-räumlichen Verfassung von Bildungsprozessen}

Der Prozesscharakter der Bildung kann auf sehr unterschiedliche Art und Weise analysiert und sozialwissenschaftlich oder philosophisch konzeptualisiert werden (vgl. KOLLER, 2012). Wie immer diese Konzepte im Einzelnen aussehen, alle verweisen mehr oder weniger eindeutig darauf, dass es sich bei der Bildung um ein fundamental zeitlich verfasstes Phänomen handelt. "Bildung und Zeit 
gehören zusammen. Bildung ist eine Zeitgestalt und ohne Zeit nicht zu haben“ (DÖRPINGHAUS; UPHOFF, 2012, S. 113). Deshalb bietet es sich an, Bildung als eine temporale Form zu untersuchen. Und zwar in objektiver Hinsicht (äußere Strukturen der Zeit, Schulzeit, Ausbildungszeit etc.) als auch in subjektiver Hinsicht (Zeitempfinden etc.) und in der Verschränkung beider Ebenen.

\section{Objektive und subjektive Bildungszeiten}

Da Bildung chromatisch verfasst ist, darf man davon ausgehen, dass VeränderungenderäußerenKoordinatenvonBildungsprozessen, derZeitordnungen, den Gehalt von Bildung nicht unberührt lassen. Das gilt es bei der Akzeleration von Schul- oder Studienzeiten, bei vorzeitigen Einschulungen, der Verlängerung von Ausbildungswegen oder bei der Umstellung von Halbtags- auf Ganztagsschulen zu bedenken und empirisch zu erforschen. Simple Kausalitäten darf man hier nicht erwarten. Aus qualitativen Studien über hochbegabte Schülerinnen und Schüler wissen wir beispielsweise, wie uneinheitlich die vermeintlich schnellen Lerner auf langwierigen oder beschleunigten Unterricht reagieren (vgl. HOYER, 2014).

Die objektiv gegebenen Bildungszeiten treffen auf innere, subjektive Zeitempfindungen und auf differente Modalitäten der Aneignung, Verarbeitung, Gestaltung und Verwirklichung von Zeit. Das Tempo und der Rhythmus, in dem Bildungsprozesse vollzogen werden, können von Individuum $\mathrm{zu}$ Individuum unterschiedlich sein, ja selbst innerhalb eines Individuums verlaufen Bildungsvorgänge mal schneller und mal langsamer. In einem Bereich ist man ein Spätzünder, anderes fliegt einem nur so zu, und wieder anderes lässt sich auch nach mehrmaliger Wiederholung nicht in die Persönlichkeit integrieren.

\section{Kein allgemeines Zeitmaß der Bildung}

Mit einem Wort: Es gibt kein allgemeingültiges Zeitmaß der Bildung. Darüber täuschen die standardisierten Bildungszeiten hinweg. Eine den individuellen Bedürfnissen und Lernvoraussetzungen angemessene Bildung benötigt vielmehr flexible, differenzierte zeitliche Arrangements.

\section{Bildung im Raum}

Bildung benötigt aber nicht nur Zeit, sie benötigt ebenso Raum. BildungsRäume sind soziale Einrichtungen, Orte und Institutionen, die sich dadurch von anderen Räumen auszeichnen, dass sie persönliche und kollektive Entwicklungen, 
Lernvorgänge, reflexive Auseinandersetzungen und Identitätsarbeit anregen und fördern.

\section{Schularchitektur}

Das findet z.B. - aber längst nicht ausschließlich (eigentlich ist prinzipiell jeder von Menschen belebte Raum ein Bildungs-Raum) - in der Schule und Hochschule statt. In der Schulhausarchitektur manifestiert sich geradezu die Bildungsauffassung einer Zeit. Denken Sie an die massiven Prachtbauten des deutschen Kaiserreichs und vergleichen Sie damit die funktionellen Schulkomplexe aus den siebziger Jahren des letzten Jahrhunderts. Denken Sie an die strengen Schulreihen früherer Jahrzehnte, wo alle Schüler einen direkten Blick nach vorne hatten, zum Lehrer - der umgekehrt die ganze Klasse mit einem Blick erfassen konnte. Und vergleichen Sie damit die variablen Inneneinrichtungen in heutigen Klassenzimmern, die, zum Teil jedenfalls, eine andere Sprache des Raumes sprechen.

Dass die unterschiedlichen Außen- wie Innenarchitekturen bei den Schülerinnen und Schülern unterschiedliche Erlebnisformen wachrufen und symbolische Botschaften vermitteln, die nicht wirkungslos bleiben, leuchtet ein (vgl. LÖW, 2001, S. 231ff.). Nicht umsonst wird dem Raum nachgesagt, er sei neben den Mitschülern und den Lehrern der dritte Erzieher. Allerdings gibt es noch nicht viele seriöse empirische Untersuchungen, die den Zusammenhang von Raum und Bildung aufschlüsseln.

\section{Bildung als (An)Ordnung}

Wie bei der Zeit, so haben wir auch beim Raum analytisch zu unterscheiden zwischen einer objektiven und einer subjektiven Seite. Autorinnen wie Martina Löw argumentieren, dass Räume generell konstituiert werden, also niemals schlichtweg physisch gegeben sind. "Raum ist eine relationale (An)Ordnung sozialer Güter und Menschen (Lebewesen) an Orten" (LÖW, 2001, S. 224). Die Konstitution von Räumen geschieht durch Synthese und Spacing.

Wie Raum konstituiert wird, ist Ergebnis eines Bildungsprozesses. Schüler oder Schülerinnen müssen lernen, institutionalisierte Verknüpfungsordnungen von sozialen Gütern und Menschen als Räume zu erkennen und dabei eigene Handlungsfähigkeiten zu entwickeln. Wie dieser Proze $\beta$ verläuft, welche Ressourcen dabei eingesetzt werden, muß weiteren Untersuchungen vorbehalten bleiben (ebd., S. 245f.). 
Für die Bildungsforschung wäre an solchen Untersuchungen besonders attraktiv, dass sie die an sich schwer fassbaren Prozesse der Bildung in ihrer räumlichen Gestalt sichtbar machen könnten.

\section{Komplexität von Bildung}

Aus diesen kurzen Bemerkungen zur zeit-räumlichen Verfassung von Bildung wird deutlich, dass es sich bei der Bildung um eine hochkomplexe Angelegenheit handelt. Subjektive und objektive, intendierte und nicht-intendierte, rationale und emotionale, reflexive und intuitive, psychologische und physiologische, individuelle und gesellschaftliche Aspekte greifen dabei ineinander. Sie können sich ergänzen, verstärken, aber auch blockieren. Aufgrund dessen sind Prognosen über den Verlauf von Bildungsprozessen schwierig, wenn nicht gar unmöglich.

\section{Grenzen der Vorhersagbarkeit und Steuerbarkeit}

Auch der gezielten Einflussnahme auf Bildungsvorgänge sind dadurch Grenzen gesetzt. Das kann für alle, die privat oder professionell mit der Bildung befasst sind, mitunter zum Verzweifeln sein. Aber im Grunde genommen ist es ein durchaus beruhigender Gedanke, dass es Erziehung, Bildung und Ausbildung mit der Widerstandskraft, Unberechenbarkeit und Eigenlogik der Akteure zu tun haben, die sich nicht nach Gutdünken verplanen, beeinflussen und steuern lassen.

\section{Erziehung - Bildung - Ausbildung}

Jetzt habe ich von Erziehung, Bildung und Ausbildung gesprochen: bedeutungsnahe, aber nicht bedeutungsidentische Ausdrücke. Wie sind sie zu unterscheiden?

\section{Erziehung}

Von Erziehung spricht man gemeinhin, wenn Einflusnahmen auf die Persönlichkeitsentwicklung anderer Personen beabsichtigt sind, wobei die Erziehungsabsicht, die Erziehungsmotivation und der Erziehungseffekt keineswegs übereinstimmen müssen. Erziehung (zumindest die Fremderziehung) endet in der Regel mit der Mündigkeit, der Selbstständigkeit und Selbstverantwortung der ZuErziehenden (vgl. EIDAM; HOYER, 2006). Bildung endet hier nicht. 
Man wird erzogen

Man spricht von Erwachsenenbildung, aber nicht von Erwachsenenerziehung. Wir nennen Schulen und Hochschulen Bildungseinrichtungen und nicht Erziehungsanstalten. Bei Erziehung liegt der Akzent aufseiten der Erziehenden, die etwas mit den Kindern oder Jugendlichen, den Zöglingen, wie es früher hieß, anstellen. Erzieherisches Handeln setzt zumeist eine asymmetrische Beziehung voraus, eine Differenz zwischen den Interaktionspartnern. Die Erziehenden sind hierbei die aktiven Personen, und die Zu-Erziehenden die eher passiven, die Empfangenden. So will es das traditionelle oft geradezu technologische Erziehungsverständnis, das aber auch noch in jüngeren Vorstellungen von sagen wir anti-autoritärer oder kommunikativer Erziehung sichtbar ist. Allerdings wird heutzutage sehr viel stärker als noch im 18. oder 19. Jahrhundert die Eigenaktivität der Zu-Erziehenden berücksichtigt.

\section{Man bildet sich selbst}

Bei der Bildung dagegen verlagert sich der Akzent. Man wird nicht gebildet (passiv), sondern man bildet sich selbst (aktiv). Dies geschieht mal mit, mal ohne Unterstützung anderer, doch immer so, dass das Moment der Selbsttätigkeit, des Sich-Bildens, im Vordergrund steht. Bildungsveranstaltungen bauen auf die prinzipielle Fähigkeit und Bereitschaft der Einzelnen, sich weiterzuentwickeln, zu lernen, sich aus- und fortzubilden.

Aus- und Fortbildung als Teilbereich der Bildung?

Dem landläufigen Sprachgebrauch nach handelt es sich bei der Aus- und Fortbildung nicht um Bildung im umfassenden Sinne, sondern um einen auf den Arbeitsmarkt und das Berufsleben zugeschnittenen Teilbereich.

Die Aus- und Fortbildung ist in Deutschland ein Ableger der Allgemeinbildung bzw. der allgemeinbildenden Schulen. Im letzten Drittel des 19. Jahrhunderts entstand mit der allgemeinen Fortbildungsschule der unmittelbare organisatorische Vorläufer der Berufsschule, wie wir sie in Deutschland bis heute kennen.

Die Fortbildungsschule war ihrerseits ein Abkömmling der Volksschule, gedacht als eine Ergänzungsinstitution für die bereits im Arbeitsleben stehenden Jugendlichen. Dass die etwa vierzehnjährigen Jungen, die nach der Volks- oder Elementarschule in die Arbeitswelt eintraten, noch bildungsbedürftig waren, stand außer Frage. Die Pioniere der ersten Fortbildungsschulen haben sich dafür 
eingesetzt, dass diese Schulen ihren Anspruch auf Bildung im umfassenden Sinne beibehielten. So erklärte der Leipziger Pädagoge Julius Kirchhoff 1879: „Wenn von Fortbildung der Volksjugend die Rede ist, so muß nach unserer Meinung zunächst daran gedacht werden, wie die Gesamtbildung des aus der Volksschule entlassenen Schülers weitergeführt werden könne“ (zit. n. BLANKERTZ, 1969, S. 130).

\section{Bildungswert von Arbeit und Beruf}

Das war der erklärte Anspruch. Die Fortbildungsschulen konnten ihn jedoch nicht erfüllen. Nach mehreren Reformen und Umstrukturierungen lief die Entwicklung schließlich auf die Entstehung der Berufsschule zu, mit der ab 1920 das duale System in Deutschland Einzug hielt. Man kann in den berufspädagogischen Dokumenten der Zeit sehr genau verfolgen, wie die Befürworter dieser Einrichtungen versuchten, den Bildungswert von Arbeit und Beruf ins Bewusstsein der Öffentlichkeit zu heben. Berufsbildung sei die „Pforte zur Menschenbildung", heißt ein berühmtes Wort von Georg Kerschensteiner. Damit protestierte er gegen die Geringschätzung der berufsorientierten Bildung, wie sie die klassischen Bildungstheoretiker Deutschlands in der Nachfolge Wilhelm v. Humboldts zumeist vertreten haben.

Der Münchner Stadtschulrat und Pädagoge Kerschensteiner trat dafür ein, dass Beruf und Arbeit konkrete Gegenstandbereiche darstellen, die ein sachbezogenes, praktisch-problemorientiertes und soziales Lernen ermöglichten, Bildungsformen also, die in den Schulen und Hochschulen viel zu kurz kamen und, wie man hinzufügen muss, immer noch kommen.

\section{Wozu Bildung? Bildung als Bürgerrecht}

Damit sind wir bei der Frage angelangt, welchem Zweck Bildung eigentlich dienen soll. Wozu Bildung?

Bildung ohne Zweck und Nutzen?

Vergegenwärtigt man sich das „klassische“ Verständnis des Bildungsbegriffs, dann kann einem die Frage „Wozu Bildung?“, wie ein Affront, ein Verrat an der Ausgangsidee von Bildung vorkommen. Muss denn die Bildung überhaupt zu irgendetwas dienlich sein, also einen Zweck haben? Die Bildungsidee der Goetheund Humboldtzeit entstand ja gerade aus dem Protest gegen das (wie man damals annahm) einseitige und utilitaristische Zweckdenken der Aufklärung. 
Neuhumanistische Bildungsidee

Den Begründern der sog. neuhumanistischen Bildungsvorstellung galten nicht Fortschritte in der materiellen oder sozialen Welt als höchstes Entwicklungsziel, sondern die Humanität, also ein moralisches und ästhetisches Ideal personaler Vollkommenheit. Dementsprechend sollte es für den Einzelnen nicht primär auf berufliche Tüchtigkeit und ökonomische Erfolge ankommen, sondern auf eine "ganzheitliche“ Persönlichkeitsentwicklung, das heißt auf die ausgewogene Entfaltung der Anlagen, Kräfte und Begabungen.

Das Vokabular aus dem Geiste Goethes, Herders, Humboldts und Schillers scheint mit den handfesten Problemen der heutigen Bildungswirklichkeit wenig zu tun zu haben. Vieles an der neuhumanistischen Bildungskonzeption wirkt in der Tat idealistisch überzeichnet, theologisch überhöht, weltfremd.

\section{Widerstand gegen die gesellschaftliche Instrumentalisierung der Bildung}

Und dennoch sollte man nicht gleich abwinken. Der Neuhumanismus postulierte Bildung als „wahren Zweck des irdischen Daseins“ - wie W. v. Humboldt sagte -, um sie vor bildungsfremden Zugriffen zu schützen. Namentlich vor der Instrumentalisierung durch Politik, Kirche und Wirtschaft. Diese drei gesellschaftlichen Kräfte stritten sich mit Beginn des bürgerlichen Zeitalters, also im 19. Jahrhundert, um die Bildungshoheit, weil sie sich von einer nach ihren Vorstellungen gebildeten Bevölkerung eine Stärkung des eigenen Systems erwarteten (vgl. HOYER, 2015).

\section{Bildung im Interessenstreit}

Vereinfacht gesagt: Der Staat wollte gehorsame Untertanen, die Kirche gottesfürchtige Menschen und die Volkswirtschaft hatte nur dann ein Interesse an der (Volks-)Bildung, wenn sich diese monetär auszahlte. Bildung als der Inbegriff des Humanen drohte auf der Strecke zu bleiben.

\section{Verantwortung der Bildungspolitik}

Nun wird man vom ökonomischen System nichts anderes erwarten, als dass es (damals wie heute) vornehmlich wirtschaftliche Zuwächse im Auge hat. Auch der Kirche wird man es nicht verübeln, wenn sie in erster Linie auf Frömmigkeit und Glaubensfestigkeit Wert legt. Von der Politik jedoch und von Volksvertretern 
in einem demokratischen Rechtsstaat allemal (der war damals ja noch in weiter Ferne), darf man sehr wohl erwarten, dass sie sich für Bildungsbedingungen stark machen, die jedem Einzelnen und der Gesellschaft insgesamt zugute kommen.

\section{Bildung ist Bürgerrecht}

Warumsolltensiedastun?WeilBildungeinBürgerrechtist.Imdeutschsprachigen Diskurs hat diesen Gedanken insbesondere Ralf Dahrendorf eingebracht. In seinem erstmals 1965 publizierten Buch „Bildung ist Bürgerrecht“ hat er das Recht auf Bildung als ein Recht aller Bürger/innen auf soziale und politische Teilhabe innerhalb einer pluralen, freien und demokratischen Gesellschaft definiert. Der Clou seiner Argumentation besteht darin, dass er sich nicht mit der formalen Gewährung von Chancengleichheit zufrieden gibt. Formale Chancengleichheit kommt den ohnedies privilegierten Gruppen zugute. Gerade für benachteiligte soziale Schichten reicht es aber nicht, wenn sie auf dem Papier die objektiven Möglichkeiten erhalten, an höherer Bildung, Kultur und Demokratie frei und individuell zu partizipieren. Sie sind dazu eben nur eingeschränkt in der Lage (das ist ja ihre Benachteiligung), solange ihnen die subjektiven Möglichkeiten fehlen, also das Verständnis, der Wunsch und Wille, die partizipatorischen Freiheiten, wie Amartya Sen (2000) das nennt, tatsächlich zu nutzen. Um in dieser Hinsicht Bildungsfortschritte in die Wege zu leiten, nimmt Dahrendorf die Gesamtgesellschaft in die Pflicht, insbesondere sieht er Aufgaben für eine „aktive Bildungspolitik“ (DAHRENDORF, 1966).

\section{Eine Art Präambel}

Auf eine überschaubare Formel gebracht stehen nach meinem Verständnis Politik und Bildungsträger in der Verantwortung, Bildungsmöglichkeiten zu schaffen, die dazu geeignet sind, erstens, dass die Einzelnen ein für sie emotional befriedigendes und vor anderen vertretbares Leben führen können; zweitens, dass sie demokratiefähige (Welt-)Bürger/innen werden und bleiben und drittens, dass sie verantwortungsbewusst, tolerant und kritisch an den gesellschaftlichen und kulturellen Vorgängen teilzunehmen vermögen.

\section{Zielkategorien von Bildung: Glück und Gerechtigkeit}

In äußerster Pointierung ließen sich diese drei Elemente sogar auf ein einziges Begriffspaar bringen, das eine Antwort auf das Wozu der Bildung verstanden werden kann. Die beiden Zielkategorien lauten: Glück und Gerechtigkeit. 


\section{Glück als Bildungskategorie}

Glück, dieses flatterhafte Ding, soll eine Antwort auf das Wozu der Bildung sein? Das hängt vom Glücksverständnis ab. Unter Glück ist hier nicht das episodische Hochgefühl gemeint. Vielmehr verstehe ich unter Glück, in Anlehnung an die antike Bedeutung des Wortes eudaimonia, ein erfülltes, insgesamt als sinnvoll empfundenes Leben, das von der jeweiligen Person bejaht und selbstverantwortlich gestaltet wird (vgl. HOYER, 2005, 2007).

\section{Glück eines gelingenden Lebens}

Für das Glück eines gelingenden Lebens lassen sich keine allgemeingültigen Inhalte anführen, da hier die individuellen und kulturellen Differenzen zu Buche schlagen. Das Glück ist auch nicht lehr- und lernbar, wie manche behaupten, da es kein unmittelbares learning outcome ist (vgl. HOYER, 2011). Aber es liegen vernünftige Vorschläge auf dem Tisch, die formale Voraussetzungen benennen, die die Gestaltung eines glücklichen Lebens, vorsichtig ausgedrückt, begünstigen.

\section{Formale Voraussetzungen eines glücklichen Lebens}

Welche Voraussetzungen sind das? Der Philosoph Martin Seel (1995) spricht von weltoffener Selbstbestimmung, von relativer Sicherheit, relativer Freiheit und relativer Gesundheit. Sind diese vier Bedingungen erfüllt, dann können sich die Individuen in der Gestaltung ihres Lebens eigenverantwortlich den vier wesentlichen Praxisfeldern zuwenden, und diese Praxisfelder sind, ebenfalls nach Seel: Arbeit, Interaktion, Spiel und Kontemplation.

\section{Pädagogische Aufgaben}

Es springt ins Auge, dass hier für die Pädagogik anspruchsvolle Aufgaben in Hülle und Fülle bereitstehen: von der Förderung von Weltoffenheit und Autonomie, über die Gewährleistung geschützter, freiheitlicher und salutogener Verhältnisse bis hin zur differenzierten Unterstützung von Arbeits-, Kommunikations-, Spielund Betrachtungsformen. Eine an diesen Elementen orientierte Bildungspraxis würde sich um das gelingende Leben der Einzelnen verdient machen, ohne sich anzumaßen, die Menschen dadurch zwangsläufig glücklich zu machen. 


\section{Gerechtigkeit setzt Grenzen}

Mit dem Streben nach Glück verhält es sich ähnlich wie mit den Spielräumen der Freiheit: Ihre Außengrenzen liegen dort, wo sie das Glück (bzw. die Freiheit) der anderen beeinträchtigen. Das ist ein ethisches Minimalprinzip, mit dem der Fokus vom Subjekt und dessen Vorstellungen vom guten Leben auf die Gemeinschaft, die Wahrung der Interessen anderer und die Gestaltung eines für alle Menschen (und Lebewesen) verträglichen Umgangs gelenkt wird.

Einer bis in die Antike zurückreichenden und wie ich finde überzeugenden Tradition zufolge gebührt dem Prinzip und der Handlungsform der Gerechtigkeit der oberste Platz in der ethischen Werte- und Tugendskala (vgl. HOYER, 2005, 2006a). Der Wille und die Fähigkeit, gerecht zu urteilen und zu handeln, sind eine unverzichtbare Bedingung, damit gerechte Gesellschaftsstrukturen eingeführt und erhalten bleiben. Und umgekehrt sind gerecht organisierte Verhältnisse das beste Bildungsmilieu zur Stärkung und Verfeinerung des Gerechtigkeitsempfindens darin stimmen die empirischen Untersuchungen von Lawrence Kohlberg, Thomas Lickona, Fritz Oser und Georg Lind überein.

\section{Gerechtigkeitsbildung}

Heranwachsende erwerben und Erwachsene erhalten sich die Tugend oder personale Kompetenz der Gerechtigkeit, insofern sie möglichst kontinuierlich die Gelegenheit bekommen, an demokratisch organisierten Lebens- und Lernwelten aktiv teilzunehmen. Zudem sollten sie Achtung, Empathie und Gerechtigkeit am eigenen Leib erfahren, zur moralischen Reflexion aufgefordert werden und dabei erleben, dass ihre Meinung gehört, ihre Handlungsmotive ernst genommen und ihre Gefühle und Affekte wertschätzend beachtet werden (HOYER, 2006b).

\section{Glück der Bildung}

Wenn den Heranwachsenden in Schulen und Hochschulen ausreichend Zeit und Raum für solche Erfahrungen zur Verfügung gestellt werden, dann erhöht sich auch die Wahrscheinlichkeit, dass den Lernenden (und Lehrenden) ein Glück zuteil wird, das den Namen Bildung verdient. 


\section{Literatur}

BLANKERTZ, Herwig. Bildung im Zeitalter der großen Industrie. Hannover: Hermann Schroedel, 1969.

DAHRENDORF, Ralf. Bildung ist Bürgerrecht. Hamburg: Nannen-Verlag, 1966.

DÖRPINGHAUS, Andreas; UPHOFF, Ina Katharina. Die Abschaffung der Zeit. Darmstadt: Hardcover, 2012.

EIDAM, Heinz; HOYER, Timo (Hg.). Erziehung und Mündigkeit. Berlin: Lit, 2006.

HOYER, Timo. Pädagogische Verantwortung für ein gelungenes Leben oder: Vom "Glück“ in der Pädagogik. In: BURCKHART, H.; SIKORA, J.; HOYER, T. Sphären der Verantwortung. Münster: Lit, 2005. S. 151-210.

HOYER, Timo. Die notwendigste Tugend. Gerechtigkeitserziehung in der Moderne. In: MILLERKIPP, G.; ZYMEK, B. (Hg.). Politik in der Bildungsgeschichte. Bad Heilbrunn: Klinkhardt, 2006a. S. 281-296.

HOYER, Timo. Gerechtigkeitserziehung und Mündigkeit in der Demokratie. In: EIDAM, H.; HOYER, T. (Hg.). Erziehung und Mündigkeit. Berlin: Lit, 2006b. S. 193-216.

HOYER, Timo. Glück und Bildung. In: HOYER, T. (Hg.). Vom Glück und glücklichen Leben. Göttingen: Vandenhoeck \& Ruprecht, 2007. S. 222-242.

HOYER, Timo. Glück soll lernbar sein? Ist es aber nicht! In: HOYER, T. et al. (Hg.). Jenseits des Individuums. Göttingen: Vandenhoeck \& Ruprecht, 2011. S. 246-260.

HOYER, Timo. Ich verstehe es halt so schnell und dann kann es auch schneller gehen. Hochbegabte in der Beschleunigungsgesellschaft. In: HOYER, T. et al. (Hg.). Sozio-Emotionalität von hochbegabten Kindern. Weinheim und Basel: Beltz, 2014. S. 124-166.

HOYER, Timo. Sozialgeschichte der Erziehung. Darmstadt: Wissenschaftliche Buchgesellschaf, 2015.

KOLLER, Hans-Christoph. Bildung anders denken. Stuttgart: Stuttgart Kohlhammer, 2012.

KOSELLECK, Reinhart. Begriffsgeschichten. Frankfurt am Main: Suhrkamp, 2010.

LÖW, Martina. Raumsoziologie. Frankfurt am Main: Suhrkamp, 2001.

SEEL, Martin. Versuch über die Form des Glücks. Frankfurt am Main: Suhrkamp, 1995.

SEN, Amartya. Ökonomie für den Menschen. München; Wien: Carl Hanser, 2000. 